\title{
Local staging with multiparametric MRI in daily clinical practice: diagnostic accuracy and evaluation of a radiologic learning curve
}

\author{
B. H. E. Jansen ${ }^{1}$ - F. H. K. Oudshoorn ${ }^{1}$ - A. M. Tijans ${ }^{1}$ - M. J. Yska ${ }^{2}$ - A. P. Lont ${ }^{3}$ - E. R. P. Collette ${ }^{2}$ J. A. Nieuwenhuijzen ${ }^{1}$. \\ A. N. Vis ${ }^{1}$
}

Received: 20 December 2017 / Accepted: 5 April 2018 / Published online: 21 April 2018

(c) The Author(s) 2018

\begin{abstract}
Purpose To estimate the diagnostic accuracy of multiparametric MRI (mpMRI) for the detection of locally advanced prostate cancer (T-stage 3-4) prior to radical prostatectomy, in a multicenter cohort representing daily clinical practice. In addition, the radiologic learning curve for the detection of locally advanced disease is evaluated.

Methods Preoperative mpMRI findings of 430 patients (2012-2016) were compared to pathology results following radical prostatectomy. The diagnostic accuracy (sensitivity, specificity, PPV, and NPV) for the detection of locally advanced disease was calculated and compared for all years separately, to evaluate the presence of a radiological learning curve.

Results Of all 137 patients with locally advanced disease, 62 patients were preoperatively detected with mpMRI [sensitivity $45.3 \%$ (95\% CI 36.9-53.6\%), specificity 75.8\% (CI 70.9-80.7\%), PPV 46.6\% (CI 38.1-55.1\%), and NPV 74.7\% (CI 69.8-79.7\%)]. The diagnostic accuracy did not improve significantly over time (sensitivity $p=0.12$; specificity $p=0.57$ ).

Conclusions In daily clinical practice, the diagnostic accuracy of mpMRI for the detection of locally advanced prostate cancer remains limited. It, therefore, seems questionable whether mpMRI is adequate to guide preoperative decision-making. No significant radiologic learning curve for the detection of locally advance disease was observed.
\end{abstract}

Keywords Prostatic neoplasms $\cdot$ Magnetic resonance imaging $\cdot$ Neoplasm staging $\cdot$ Learning curve

B. H. E. Jansen

bh.jansen@vumc.nl

F. H. K. Oudshoorn

frederikoudshoorn@gmail.com

A. M. Tijans

alinetijans@gmail.com

M. J. Yska

yskam@maasstadziekenhuis.nl

A. P. Lont

annelont@yahoo.com

E. R. P. Collette

eelco@collette.nl

J. A. Nieuwenhuijzen

j.nieuwenhuijzen@vumc.nl

A. N. Vis

a.vis@vumc.nl

1 VU University Medical Center, Amsterdam, The Netherlands

2 Maasstad Ziekenhuis, Rotterdam, The Netherlands

3 Meander Medisch Centrum, Amersfoort, The Netherlands

\section{Introduction}

Prostate cancer $(\mathrm{PCa})$ is the most common cancer in men of older age in Western countries [1]. Accurate staging of the primary tumor is of vital importance, as the distinction between organ-confined disease (T-stage $1-2$ ) versus locally advanced tumors (T3-4) influences both prognosis [2] and treatment planning [3].

The main therapeutic approaches for PCa include radical prostatectomy and radiotherapy [3]. When considering a radical prostatectomy, the presence of locally advanced disease warrants a concomitant extended pelvic lymph node dissection (ePLND), as there is an increased risk of lymph node metastasis [3-5]. In addition, local tumor stage guides surgical planning regarding the preservation of the neurovascular bundle. Nerve-sparing surgery is generally restricted to patients with organ-confined disease. Extension of PCa outside the prostatic capsule requires dissection of the neurovascular bundle, for nerve-sparing surgery would increase the risk of positive surgical margins $[3,4,6]$. The assessment of the local tumor stage is similarly important 
when radiotherapy is chosen as treatment and guides decisions on radiation dose, radiation template, and adjuvant therapies [3, 7].

For assessment of the local tumor stage, routine diagnostics (i.e., digital rectal examination, serum prostate-specific antigen (PSA) level, transrectal ultrasound, and biopsy Gleason score [3, 8]) are insufficient [9]. When combining these clinical parameters into predictive nomograms (e.g., the Partin Tables), staging accuracy increases, but remains imperfect [10, 11].

To overcome this diagnostic shortcoming, multiparametric magnetic resonance imaging (mpMRI) is increasingly deployed. mpMRI is an imaging technique that combines different (functional) imaging sequences, generating improved detection, and localization of malignant lesions. Although mpMRI presents promising detection of $\mathrm{PCa}$ [12], accurate assessment of the tumor stage is still imperfect. In a recent meta-analysis, the sensitivity of mpMRI for overall T3 detection reached $61 \%$ only (95\% CI 54-67\%) [13].

A concern regarding mpMRI is the considerable interobserver variability $[14,15]$. This problem might be due to different experience of radiologists with mpMRI, as a marked radiologic learning curve was demonstrated [16-19]. The presence of such learning curve, however, is studied mainly for primary detection of PCa. Research specifically evaluating the existence of a radiological learning curve for correct staging of $\mathrm{PCa}$ is scare, focusing mainly on endorectal MRI [18]. In the cited meta-analysis, the effect of radiologists' experience on diagnostic accuracy was evaluated, but the results were inconclusive [13].

In this study, we aimed to estimate the diagnostic accuracy of mpMRI for the detection of locally advanced $\mathrm{PCa}$ stages (pT3-4) prior to radical prostatectomy, in a multicenter, real-life clinical cohort of patients. We additionally assessed the diagnostic accuracy over time, evaluating the existence of a radiologic learning curve.

\section{Materials and methods}

\section{Subjects}

For this study, 430 concurrent patients were retrospectively analyzed. Inclusion criteria were histologically confirmed prostate adenocarcinoma, for which a robot-assisted laparoscopic radical prostatectomy (RARP) and preoperative mpMRI were performed. Both MRI acquisitions made before or after prostate biopsy were considered eligible for inclusion, as in both scenarios, staging information is provided. The indication to perform an RARP as well as an mpMRI was made according to the locally valid clinical guidelines $[3,20]$. These guidelines recommend mpMRI for intermediate- and high-risk patients [3] and when "clinically relevant for therapy planning'-explicitly mentioning decisions regarding nerve-sparing surgery [20]. In what exact scenarios an mpMRI is clinically relevant is left to the urologists' discretion.

Patients were included from 2012 until 2016, in three hospitals in The Netherlands (VU University Medical Center, Amsterdam; Maasstad Ziekenhuis, Rotterdam; Meander Medisch Centrum, Amersfoort). For all participants, demographic and clinical data were retrieved (e.g., age, clinical stage, prostate biopsy results, and recent PSA).

\section{Imaging protocol and analysis}

All institutions used three Tesla MRI scanners $\left(\mathrm{GE}^{\circledR}\right.$, Siemens ${ }^{\circledR}$ ). The imaging protocol included T1-weighted, T2-weighted, diffusion weighed, and dynamic contrastenhanced imaging. No endorectal coils were used. Per hospital, mpMRI interpretation was done by two to three radiologists dedicated to prostate mpMRI reading. As our series report on staging in daily clinical practice, the radiologists were not blinded to available clinical information and revisions of mpMRI acquisitions from referred patients were not standardly performed. During the course of this study, the use of standardized reporting for mpMRI became in use (PI-RADS v1 [21]; PI-RADS v2 [22]), providing guidelines for assigning rT3-4 stages on mpMRI.

\section{Pathologic analysis}

RARP specimens were processed according to clinical routine [3] in the participating hospitals by dedicated uropathologists. No centralized review of the analyses was performed. Specimens were fixated with formaldehyde (10\%) and the apex and base removed. The mid part of the specimen was cut perpendicular to the urethra in $4 \mathrm{~mm}$ slices; the apex and base were cut in sagittal fashion. The resulting slices were processed after sectioning in quadrants. Pathology reporting included histopathologic cancer type, Gleason score, and explicit notation of the presence or absence of any form of local tumor advancement (pT3a, pT3b, and pT4).

\section{Statistics}

Overall detection of malignancy was calculated (sensitivity). When a PI-RADS classification was given, scores 4 and 5 were considered a positive test result. Radiological T-stage (rT) based on mpMRI was compared to the pathological T-stage (pT). Sensitivity, specificity, positive predicting value (PPV), and negative predicting value (NPV) of mpMRI were calculated for locally advanced disease (pT3-4). To examine if the accuracy of mpMRI was different in patients with a high risk of locally advanced disease, all patients with a Gleason score $\geq 8$ and/or a PSA of 
$\geq 20 \mathrm{ng} / \mathrm{ml}$ were identified. The diagnostic accuracy in this high-risk group was compared to the accuracy in the lower risk group (Gleason score 6-7; PSA less than $20 \mathrm{ng} / \mathrm{ml}$ ).

Finally, the diagnostic accuracy was analyzed for all years (2012-2016) separately, to evaluate the presence of a radiological learning curve. To overcome small sample sizes per

Table 1 Characteristics of included patients

\begin{tabular}{ll}
\hline Patient characteristics and pathology results & \\
\hline Age (years) & $66(61-69)$ \\
PSA (ng/ml) & $9.2(6.2-14.9)$ \\
Prostate volume (ml) & $48(37-65)$ \\
Number of biopsy cores & $8(8-10)$ \\
$\%$ of positive biopsy cores & 49.1 \\
Gleason score (biopsy) & \\
6 & $156(36 \%)$ \\
7 & $182(42 \%)$ \\
8 & $59(14 \%)$ \\
9 & $27(6 \%)$ \\
10 & $5(1 \%)$ \\
Pathology (prostatectomy specimens) & \\
pT0 & $4(1 \%)$ \\
pT2a & $40(9 \%)$ \\
pT2b & $9(2 \%)$ \\
pT2c & $240(56 \%)$ \\
pT3a & $76(18 \%)$ \\
pT3b & $57(13 \%)$ \\
pT4 & $4(1 \%)$ \\
\hline
\end{tabular}

Median and interquartile ranges; number and percentages of total year, an extra analysis of diagnostic accuracy based on the first and second half of the inclusions per hospital was performed. Differences in diagnostic performance were checked for statistical significance $(p<0.05)$ using the $\chi^{2}$ test.

\section{Results}

An overview of the patients' characteristics is presented in Table 1. A PI-RADS classification was given in $60.0 \%$ of all cases (rising from $0 \%$ in 2012 to $65.1 \%$ in 2016). Pathology analysis following radical prostatectomy revealed extra-prostatic extension (pT3a) in $76(18 \%)$ patients, seminal vesical invasion in $57(13 \%)$ patients, and advancement of the tumor into adjacent structures (pT4) in $4(1 \%)$ patients.

The presence of malignancy was correctly detected by mpMRI in $n=358$ patients (sensitivity $84.0 \%$, CI 80.6-87.5\%). In Table 2, the findings on preoperative mpMRI (rT) and concurrent RARP pathology results (pT) are depicted.

mpMRI detected 62 out of 137 patients with locally advanced disease (pT3-4), resulting in a sensitivity of $45.3 \%$ (CI 36.9-53.6\%), specificity 75.8\% (CI 70.9-80.7\%), PPV 46.6\% (CI 38.1-55.1\%), and NPV of 74.7\% (CI 69.8-79.7\%). Sensitivity in the group with high risk of locally advanced disease $(n=133)$ was $49.2 \%$ (CI $36.4-61.9 \%)$ versus $42.3 \%$ (CI 31.4-53.3\%) in the lower risk group $(n=297)(p=0.49)$. Specificity was $73.0 \%$ (CI $62.9-83.1 \%$ ) and $76.3 \%$ (CI 70.6-82.0), respectively.

Radiologic understaging (i.e., the failure to detect locally advanced disease) occurred in $n=75$ cases ( $54.7 \%$
Table 2 Cross-tabulation of pathological tumor stage (pT) and radiological tumor stage (rT) for 430 patients undergoing mpMRI and robot-assisted radical prostatectomy (RARP)

\begin{tabular}{|c|c|c|c|c|c|c|c|}
\hline & \multicolumn{7}{|c|}{ Pathological tumor stage } \\
\hline & pT0 & pT2 & pT3 total & pT3a & pT3b & pT4 & Total \\
\hline \multicolumn{8}{|c|}{ Radiological tumor stage } \\
\hline \multirow[t]{2}{*}{ rT0/rTx } & 2 & 56 & 12 & 9 & 3 & $\mathbf{0}$ & 70 \\
\hline & $0 \%$ & $13 \%$ & $3 \%$ & $2 \%$ & $1 \%$ & $\mathbf{0} \%$ & $16 \%$ \\
\hline \multirow[t]{2}{*}{ rT2 } & 2 & 162 & 59 & 32 & 27 & 4 & 227 \\
\hline & $0 \%$ & $38 \%$ & $14 \%$ & $7 \%$ & $6 \%$ & $1 \%$ & $53 \%$ \\
\hline \multirow[t]{2}{*}{ rT3 total } & 0 & 70 & 62 & $\mathrm{x}$ & $\mathrm{x}$ & 0 & 132 \\
\hline & $0 \%$ & $16 \%$ & $14 \%$ & & & $0 \%$ & $31 \%$ \\
\hline \multirow[t]{2}{*}{ rT3a } & 0 & 68 & $\mathrm{x}$ & 33 & 16 & 0 & 117 \\
\hline & $0 \%$ & $16 \%$ & & $8 \%$ & $4 \%$ & $0 \%$ & $27 \%$ \\
\hline \multirow[t]{2}{*}{ rT3b } & 0 & 2 & $\mathrm{x}$ & 2 & 11 & 0 & 15 \\
\hline & $0 \%$ & $0 \%$ & & $0 \%$ & $3 \%$ & $0 \%$ & $3 \%$ \\
\hline \multirow[t]{2}{*}{ rT4 } & 0 & 1 & 0 & 0 & 0 & 0 & 1 \\
\hline & $0 \%$ & $0 \%$ & $0 \%$ & $0 \%$ & $0 \%$ & $0 \%$ & $0 \%$ \\
\hline \multirow[t]{2}{*}{ Total } & 4 & 289 & 133 & 76 & 57 & 4 & 430 \\
\hline & $1 \%$ & $67 \%$ & $31 \%$ & $18 \%$ & $13 \%$ & $1 \%$ & $100 \%$ \\
\hline
\end{tabular}

Indicated in bold are cases of radiologic understaging, with potential oncologic hazard. Indicated in italic are cases with radiologic overstaging, influencing the decision to perform nerve-sparing surgery 
of all patients with pT3-4). In $n=30$ of these patients, no ePLKD was performed (21.9\% all patients with pT3-4), and in $n=41$ patients, complete nerve preservation was performed (29.9\% of all pT3-4). This reveals the potential undertreatment associated with incorrect radiologic staging. Radiologic overstaging (incorrect detection of locally advanced disease) was present in $n=70$ cases (23.9\% of patients with pT0-2). In $n=16$ of these cases, no form of nerve-sparing was performed (5.5\% of all patients with organ-confined disease), revealing potential overtreatment.

In Fig. 1, the diagnostic accuracy of mpMRI for the detection of locally advanced disease is presented for all study years separately (radiologic learning curve). Over time, a negative trend was observed, although the differences in diagnostic accuracy were not statistically significant (sensitivity per year $p=0.12$, specificity per year $p=0.57$; sensitivity per half sample $p=0.61$ ).

\section{Discussion}

The preoperative assessment of local tumor stage affects important therapeutic decisions regarding radicality of the surgical procedure (i.e., a wide or less wide excision around the prostate; the performance of an ePLND) and the ability to preserve structures that relate to functional outcomes (i.e., to perform nerve-sparing surgery or not).

In our extensive, multicenter cohort, the accuracy of mpMRI for local tumor staging was evaluated. The reference standard was the pathology report following RARP. Intriguingly, more than half of the cases (55\%) with locally advanced disease (pT3-4) remained undetected by preoperative mpMRI (see Table 2). Conversely, if mpMRI indicated locally advanced disease, in more than half of the cases, these results proved to be falsely positive, as pathological examination showed organ-confined disease. We observed no significant increase in diagnostic accuracy

\section{Detection of pT3-4}

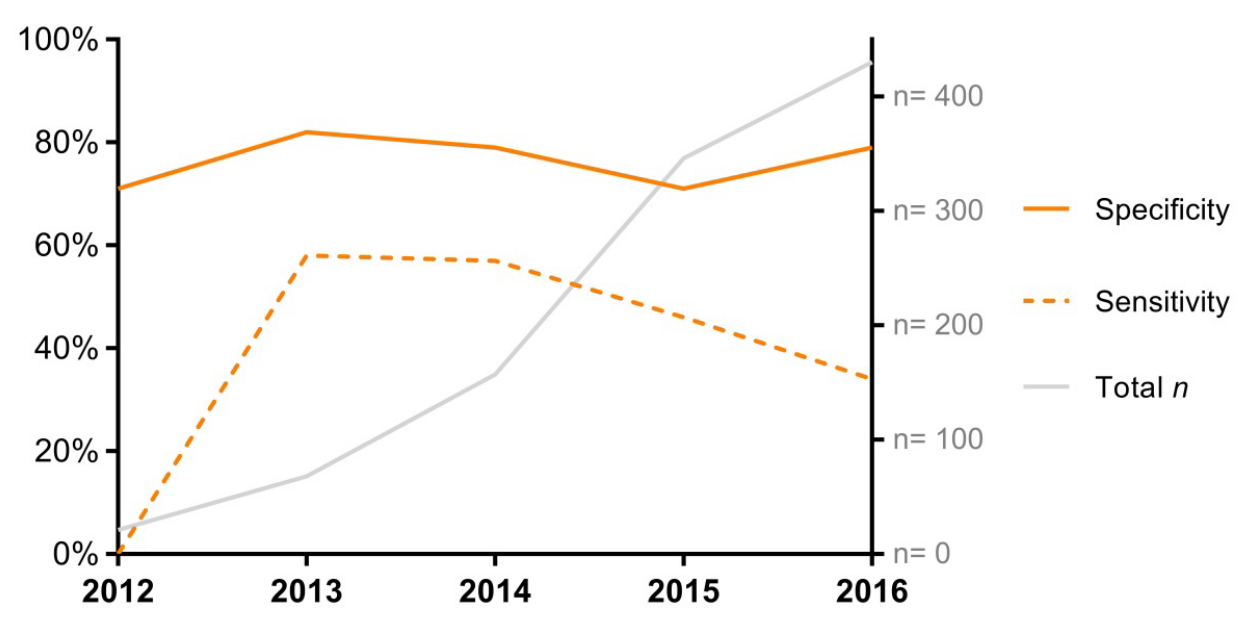

\begin{tabular}{|c|c|c|c|c|c|c|}
\hline & 2012 & 2013 & 2014 & 2015 & 2016 & Overall \\
\hline$N$ & 21 & 47 & 89 & 189 & 84 & 430 \\
\hline Prevalence of pT3-4 ${ }^{a}$ & $19 \%$ & $26 \%$ & $34 \%$ & $31 \%$ & $38 \%$ & $32 \%$ \\
\hline \multicolumn{7}{|l|}{ Detection of $p T 3-4$} \\
\hline Sensitivity & $0 \%$ & $58 \%$ & $57 \%$ & $46 \%$ & $34 \%$ & $45 \%$ \\
\hline Specificity & $71 \%$ & $83 \%$ & $80 \%$ & $72 \%$ & $79 \%$ & $75 \%$ \\
\hline PPV & $0 \%$ & $54 \%$ & $59 \%$ & $42 \%$ & $50 \%$ & $47 \%$ \\
\hline NPV & $75 \%$ & $85 \%$ & $78 \%$ & $74 \%$ & $66 \%$ & $74 \%$ \\
\hline Accuracy & $57 \%$ & $77 \%$ & $72 \%$ & $63 \%$ & $62 \%$ & $66 \%$ \\
\hline
\end{tabular}

Fig. 1 Number of included mpMRI procedures between 2012 and 2016 and the radiologic detection of locally advanced disease (pT3-4) 
when confining the use of mpMRI to patients with high risk of locally advanced disease only.

Our results are in line with those observed in the review by De Rooij et al. [13]; and taking together, it appears questionable whether mpMRI is adequate to guide therapeutic decision-making. Moreover, mpMRI does not seem superior in predicting locally advanced disease compared to routine clinical parameters such as those used in the Partin tables [11]. There may be a role for mpMRI when results are combined with clinical parameters, as a recent study showed some increased accuracy for such combination (not a predefined nomogram) [23]. Future research might clarify if the incorporation of mpMRI to the established nomograms could increase diagnostic confidence.

Novel insights of this study include the clinical impact of erroneous staging and the evaluation of a radiologic learning effect. Radiologic understaging potentially impairs oncologic outcomes, as patients might not receive the indicated ePLND or might be operated with a possibly hazardous nerve-sparing approach. We have shown that in a third of all patients with locally advanced disease, radiologic understaging might have contributed to a form of undertreatment.

Radiologic overstaging, on the other hand, leads to patients unnecessarily being withheld nerve-sparing surgery, causing impaired potency and urinary continence. In our cohort, for 16 men with organ-confined disease, this form of overtreatment seemed present (6\% of all patients with organ-confined disease).

The above-mentioned numbers should be interpreted cautiously. Due to the retrospective nature of our study, causality cannot be proven. Furthermore, no complete insight into surgical decision-making was present. For example, the choice to not perform an ePLND could have been caused by radiologic understaging, but patient factors outside the scope of this study may also have been the reason to omit an ePLND (e.g., a technically unfeasible procedure). The true extent of undertreatment, caused by inaccurate mpMRI findings, might, therefore, have been more limited.

The same applies the identified patients with potential overtreatment. We cannot know whether all of these patients would have opted for a nerve-sparing approach, if preoperative radiologic staging would have been correct. Preoperative erectile function, for example, may have been absent, rendering nerve-sparing surgery futile.

In our cohort, no radiological learning curve could be observed. Despite the rising experience with mpMRI and increasing number of performed procedures, the sensitivity for locally advanced disease did not improve over the years. In the previous reports as well, the number of included procedures had no influence on diagnostic accuracy [13], challenging the presumed gain of concentrating mpMRIs to a limited number of hospitals.
Although our sample size was substantial (430 patients), one might question whether 137 patients with locally advanced disease divided over different hospitals, over multiple years' time, were sufficient to facilitate learning. It is important to realize, however, that radiologists were exposed to more mpMRI studies than included in this analysis (i.e., also radiotherapy patients are staged with mpMRI). Besides, the included centers are all recognized reference centers for $\mathrm{PCa}$ care, meaning that these numbers comprise the realistic clinical volumes in the present-day oncologic care.

In this study, we examined the existence of a radiologic learning curve on a hospital level. We cannot formally exclude the possibility that a learning curve was present for individual radiologists, as no strict, prospective protocol was followed. However, it is frequently stated that mpMRI should be confined to expert centers [3, 24], implying general hospital-level learning. As individual radiologists may change employment, the evaluation of a learning curve irrespective of changes in individual expertise is warranted.

Our study has some additional limitations. The available information on the radiologists performing mpMRI interpretation was limited. In the included hospitals, prostate mpMRIs are strictly reviewed by dedicated radiologists. Although this is widespread practice, we cannot formally exclude the possibility that less experienced radiologists have occasionally reviewed scans in referring centers. Standardly performing a central revision of the mpMRI images might have overcome such imperfection, but is not in line with the current clinical practice.

Another point is the impact of the rising number of scans on clinical workflow. We hypothesized that such increase would lead to more radiologic experience and thereby to higher diagnostic accuracy. However, we do not know that the available time radiologists were given to review all scans. Given the marked rise in number of scans, the available time per scan could have been comprised, possibly offsetting a potential learning effect.

The strength of our analysis lies in the fair representation of clinical reality in the present-day oncologic centers. If the volumes, methods, and interpretation of mpMRI acquisitions in these acknowledged reference centers do not lead to accurate results, it seems challenging to ensure widespread value of the imaging technique.

\section{Conclusions}

In this multicenter, real-life clinical cohort of patients, the diagnostic accuracy of mpMRI for the detection of locally advanced PCa stages was limited. Therefore, it remains questionable whether the diagnostic accuracy of mpMRI is sufficient to guide therapeutic decision-making. In addition, 
no significant radiologic learning curve for the detection of locally advance disease was observed.

Author contributions B.H.E. Jansen: project development; data management; data analysis; manuscript writing. F.H.K. Oudshoorn: project development; data management; data analysis; manuscript writing. A.M. Tijans: project development; data management; data analysis; manuscript writing. M.J. Yska: project development; data management; manuscript writing. A. P. Lont: project development; manuscript writing. E.R.P. Collette: project development; data management; manuscript writing. J.A. Nieuwenhuijzen: project development; manuscript writing. A.N. Vis: project development; data management; data analysis; manuscript writing

\section{Compliance with ethical standards}

No support from any agency in the public, commercial, or not-for-profit sectors was received for this research. For this type of study, formal consent is not required.

Conflict of interest The authors declare that they have no conflicts of interest.

Open Access This article is distributed under the terms of the Creative Commons Attribution 4.0 International License (http://creativeco mmons.org/licenses/by/4.0/), which permits unrestricted use, distribution, and reproduction in any medium, provided you give appropriate credit to the original author(s) and the source, provide a link to the Creative Commons license, and indicate if changes were made.

\section{References}

1. Ferlay J, Soerjomataram I, Dikshit R, Eser S, Mathers C, Rebelo M, Parkin DM, Forman D, Bray F (2015) Cancer incidence and mortality worldwide: sources, methods and major patterns in GLOBOCAN 2012. Int J Cancer 136(5):E359-E386. https://doi. org/10.1002/ijc. 29210

2. Cooperberg MR, Pasta DJ, Elkin EP, Litwin MS, Latini DM, Du Chane J, Carroll PR (2005) The University of California, San Francisco Cancer of the prostate risk assessment score: a straightforward and reliable preoperative predictor of disease recurrence after radical prostatectomy. J Urol 173(6):1938-1942. https://doi. org/10.1097/01.ju.0000158155.33890.e7

3. Mottet N, Bellmunt J, Bolla M, Briers E, Cumberbatch MG, De Santis M, Fossati N, Gross T, Henry AM, Joniau S, Lam TB, Mason MD, Matveev VB, Moldovan PC, van den Bergh RCN, Van den Broeck T, van der Poel HG, van der Kwast TH, Rouviere O, Schoots IG, Wiegel T, Cornford P (2017) EAU-ESTRO-SIOG guidelines on prostate cancer. Part 1: screening, diagnosis, and local treatment with curative intent. Eur Urol 71(4):618-629

4. Fahmy O, Khairul-Asri MG, Hadi S, Gakis G, Stenzl A (2017) The role of radical prostatectomy and radiotherapy in treatment of locally advanced prostate cancer: a systematic review and metaanalysis. Urol Int. https://doi.org/10.1159/000478789

5. Choo MS, Kim M, Ku JH, Kwak C, Kim HH, Jeong CW (2017) Extended versus standard pelvic lymph node dissection in radical prostatectomy on oncological and functional outcomes: a systematic review and meta-analysis. Ann Surg Oncol 24(7):2047-2054. https://doi.org/10.1245/s10434-017-5822-6
6. Sokoloff MH, Brendler CB (2001) Indications and contraindications for nerve-sparing radical prostatectomy. Urol Clin North Am 28(3):535-543

7. Bolla M, Van Tienhoven G, Warde P, Dubois JB, Mirimanoff RO, Storme G, Bernier J, Kuten A, Sternberg C, Billiet I, Torecilla JL, Pfeffer R, Cutajar CL, Van der Kwast T, Collette L (2010) External irradiation with or without long-term androgen suppression for prostate cancer with high metastatic risk: 10-year results of an EORTC randomised study. Lancet Oncol 11(11):1066-1073. https://doi.org/10.1016/s1470-2045(10)70223-0

8. Donovan J, Hamdy F, Neal D, Peters T, Oliver S, Brindle L, Jewell D, Powell P, Gillatt D, Dedman D, Mills N, Smith M, Noble S, Lane A (2003) Prostate testing for cancer and treatment (ProtecT) feasibility study. Health Technol Assess 7(14):1-88

9. Schreiber D, Wong AT, Rineer J, Weedon J, Schwartz D (2015) Prostate biopsy concordance in a large population-based sample: a surveillance, epidemiology and end results study. J Clin Pathol 68(6):453-457. https://doi.org/10.1136/jclinpath-2014-202767

10. Eifler JB, Feng Z, Lin BM, Partin MT, Humphreys EB, Han M, Epstein JI, Walsh PC, Trock BJ, Partin AW (2013) An updated prostate cancer staging nomogram (Partin tables) based on cases from 2006 to 2011 . BJU Int 111(1):22-29. https://doi. org/10.1111/j.1464-410X.2012.11324.x

11. Tosoian JJ, Chappidi M, Feng Z, Humphreys EB, Han M, Pavlovich CP, Epstein JI, Partin AW, Trock BJ (2017) Prediction of pathological stage based on clinical stage, serum prostate-specific antigen, and biopsy Gleason score: partin tables in the contemporary era. BJU Int 119(5):676-683. https://doi.org/10.1111/ bju. 13573

12. Hegde JV, Mulkern RV, Panych LP, Fennessy FM, Fedorov A, Maier SE, Tempany CM (2013) Multiparametric MRI of prostate cancer: an update on state-of-the-art techniques and their performance in detecting and localizing prostate cancer. J Magn Reson Imaging 37(5):1035-1054. https://doi.org/10.1002/jmri.23860

13. de Rooij M, Hamoen EH, Witjes JA, Barentsz JO, Rovers MM (2016) Accuracy of magnetic resonance imaging for local staging of prostate cancer: a diagnostic meta-analysis. Eur Urol 70(2):233-245. https://doi.org/10.1016/j.eururo.2015.07.029

14. Rosenkrantz AB, Ginocchio LA, Cornfeld D, Froemming AT, Gupta RT, Turkbey B, Westphalen AC, Babb JS, Margolis DJ (2016) Interobserver reproducibility of the PI-RADS version 2 lexicon: a multicenter study of six experienced prostate radiologists. Radiology 280(3):793-804. https://doi.org/10.1148/radio 1.2016152542

15. Muller BG, Shih JH, Sankineni S, Marko J, Rais-Bahrami S, George AK, de la Rosette JJ, Merino MJ, Wood BJ, Pinto P, Choyke PL, Turkbey B (2015) Prostate cancer: interobserver agreement and accuracy with the revised prostate imaging reporting and data system at multiparametric MR imaging. Radiology 277(3):741-750. https://doi.org/10.1148/radiol.2015142818

16. Rosenkrantz AB, Ayoola A, Hoffman D, Khasgiwala A, Prabhu V, Smereka P, Somberg M, Taneja SS (2017) The learning curve in prostate MRI interpretation: self-directed learning versus continual reader feedback. AJR Am J Roentgenol 208(3):W92-W100. https://doi.org/10.2214/ajr.16.16876

17. Gaziev G, Wadhwa K, Barrett T, Koo BC, Gallagher FA, Serrao E, Frey J, Seidenader J, Carmona L, Warren A, Gnanapragasam V, Doble A, Kastner C (2016) Defining the learning curve for multiparametric magnetic resonance imaging (MRI) of the prostate using MRI-transrectal ultrasonography (TRUS) fusionguided transperineal prostate biopsies as a validation tool. BJU Int 117(1):80-86. https://doi.org/10.1111/bju.12892

18. Latchamsetty KC, Borden LS Jr, Porter CR, Lacrampe M, Vaughan M, Lin E, Conti N, Wright JL, Corman JM (2007) Experience improves staging accuracy of endorectal magnetic 
resonance imaging in prostate cancer: what is the learning curve? Can J Urol 14(1):3429-3434

19. Akin O, Riedl CC, Ishill NM, Moskowitz CS, Zhang J, Hricak $H$ (2010) Interactive dedicated training curriculum improves accuracy in the interpretation of MR imaging of prostate cancer. Eur Radiol 20(4):995-1002. https://doi.org/10.1007/s0033 0-009-1625-x

20. Integraal Kankercentrum Nederland, Nederlandse Vereniging voor Urologie (2016) Prostaatcarcinoom, Landelijke richtlijn, version 2016. http://oncoline.nl/prostaatcarcinoom

21. Barentsz JO, Richenberg J, Clements R, Choyke P, Verma S, Villeirs G, Rouviere O, Logager V, Futterer JJ (2012) ESUR prostate MR guidelines 2012. Eur Radiol 22(4):746-757. https://doi. org/10.1007/s00330-011-2377-y

22. Weinreb JC, Barentsz JO, Choyke PL, Cornud F, Haider MA, Macura KJ, Margolis D, Schnall MD, Shtern F, Tempany CM,
Thoeny HC, Verma S (2016) PI-RADS prostate imaging-reporting and data system: 2015, version 2. Eur Urol 69(1):16-40. https ://doi.org/10.1016/j.eururo.2015.08.052

23. Lebacle C, Roudot-Thoraval F, Moktefi A, Bouanane M, De La Taille A, Salomon L (2017) Integration of MRI to clinical nomogram for predicting pathological stage before radical prostatectomy. World J Urol 35(9):1409-1415. https://doi.org/10.1007/ s00345-016-1981-5

24. Kirkham AP, Haslam P, Keanie JY, McCafferty I, Padhani AR, Punwani S, Richenberg J, Rottenberg G, Sohaib A, Thompson P, Turnbull LW, Kurban L, Sahdev A, Clements R, Carey BM, Allen C (2013) Prostate MRI: who, when, and how? Report from a UK consensus meeting. Clin Radiol 68(10):1016-1023. https://doi. org/10.1016/j.crad.2013.03.030 